\title{
Passive mechanical properties of the wrist and physiological tremor
}

\author{
M LAKIE, EG WALSH, GW WRIGHT \\ Erom the Department of Physiology, University Medical School, Edinburgh, UK
}

SUMMARY Observations on wrist tremor have been made in the horizontal plane using a tremorograph of new design. A small tap to the hand gave rise to a decrementing series of oscillations at about $9 \mathrm{~Hz}$; as these effects were seen in relaxed and in anaesthetised subjects they were not due to neural reflexes. With the fingers partially extended the spontaneous tremor increased markedly and taps again gave rise to a series of oscillations. Under these circumstances there was no modulation of the EMG. It is accordingly concluded that mechanical wrist resonance plays a major role in the forms of tremor studied.

There are two main theories concerning the origin of physiological tremor. In the first the ripple resulting from the asynchronous unfused activity of motor units is regarded as the driving force. Rietz \& Stiles ${ }^{1}$ after tapping the hand have observed decrementing transients which they attribute to mechanical factors. They suggest that physiological tremor results from random forcing of an underdamped second order system. The muscle acts as a low pass filter. ${ }^{2}$ The second theory involves the stretch reflex. According to this theory the delays in the high gain feedback loop generate an oscillation at $8-12 \mathrm{~Hz}$. The original evidence in favour of the stretch reflex theory is an experiment of Lippold ${ }^{3}$ in which the outstretched finger was mechanically prodded. There resulted: (1) a series of mechanical oscillations at the same frequency as the tremor but of greater amplitude, and (2) modulation of the electromyogram (EMG) of the active muscle in time with these oscillations. It was concluded that the rhythmic activity of the muscle caused the oscillations. However, a $9.5 \mathrm{~Hz}$ tremor has been seen in a patient with a deafferented arm. ${ }^{4}$

In many investigations of tremor the motion of the fingers or wrist has been in the vertical plane, with the extensor muscles tonically contracted to counteract gravity. Under these conditions the passive properties of the musculature are concealed. We have argued that a satisfactory comparison of the active and passive state of the musculature can only be made in the horizontal plane with the hand supported. We have

Address for reprint requests: Prof EG Walsh, Department of Physiology, University Medical School, Teviot Place, Edinburgh EH8 9AG, UK.

Received 24 September 1985. Accepted 16 October 1985 sought to investigate the response to gentle taps which are nonetheless sufficient to generate a disturbance which is larger than the prevailing tremor; much of this work involved a tremorograph of new design. This paper describes oscillations similar to those found by Lippold but which are not accompanied by synchronised EMG activity. Furthermore, we show that these oscillatory transients can occur in conditions in which all active muscular activity is suspended. The short range resonant passive behaviour of the wrist joint and associated structures is a new finding which calls for significant modification of current theories.

\section{Methods}

Most of the recordings were made with an isotonic "hanging hand" Tremorograph (fig 1). The heart of the instrument is an induction generator (a drag cup unit) which is used as an angular accelerometer. ${ }^{5}$ In this device the moving element, a copper cylinder of $28 \mathrm{~mm}$ diameter, rotates in a magnetic field provided by one of the stator windings. The currents thus induced in the copper cylinder are picked up by the second stator winding as a voltage proportional to angular acceleration. There are no brushes and only one moving part. Friction is negligible and the inertia, when combined with the attachments required for the experiments is only $0.2 \mathrm{~g} \mathrm{~m}^{2}$, a value which is small compared with the inertia of a hand, typically $1.0-2.0 \mathrm{~g} \mathrm{~m}^{2}$. To the lower end of the shaft of the generator a light adjustable crank was attached and this in turn supported the hand by a "Velcro" sling. The suspended hand was thus free to move in flexion or extension in the horizontal plane. The forearm was supported and prevented from rotating by being taped to a vertical metal plate just proximal to the wrist joint. The position of the supporting system was adjustable so that the axis of the joint could be accurately aligned with the instrument. 


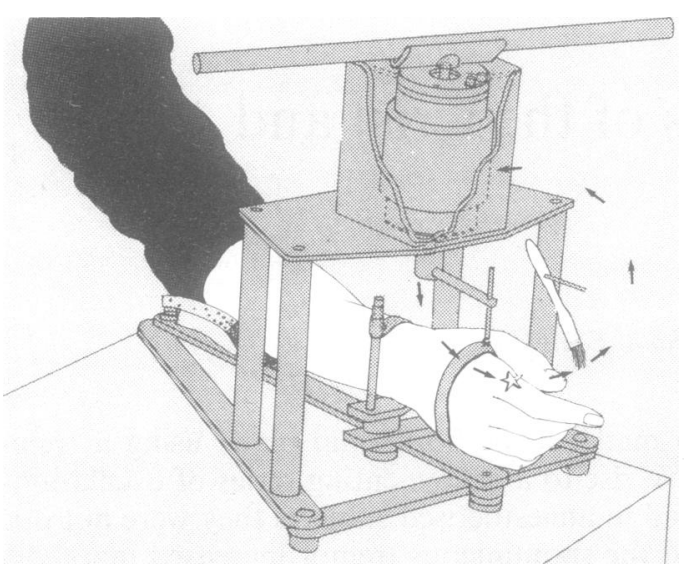

Fig 1 The hanging hand tremorograph. The cylindrical induction generator is magnetically screened with mumetal.

The effect of adding inertia may be relevant to an understanding of tremor mechanisms. Some experimenters have placed a weight on the outstretched hand. ${ }^{67}$ To maintain the same position there must therefore be a compensatory increase in voluntary motor activity. The effects of the inertial load are thus compounded by the effects of raised muscular tension and the resulting findings are likely to be confused. We argue that in such experiments the mass should be balanced. In the hanging hand tremorograph this was achieved by supporting one of a variety of steel bars centrally in an alloy fitting of $\mathrm{V}$ section attached to the upper end of the shaft. High speed macro-cinematography confirmed that even with the largest bars used the coupling between man and machine was adequately rigid. Small taps could be applied to the hand manually, but a more systemic approach was permitted by the use of an artist's brush which was rotated by a small geared motor so as to strike the hand periodically. There was a pause between strikes so that the transients induced by one tap did not run into those of the next.

For the experiments in the vertical plane the hand was attached by a light crank to a small electric motor (Philips 010) powered by a current servo amplifier. Small rectangular pulses of torque of adjustable rate, strength and duration could thus be applied to the wrist joint. The velocity of the resultant oscillations were recorded by a tachometer in tandem with the motor. All records were made on a multichannel polygraph. Surface EMG from the flexor and extensor muscles was recorded; suction cup electrodes were used. An electronic integrator supplied with one of the EMG signals was also displayed. An audio amplifier permitted subjects to maintain a reasonably constant degree of contraction when so instructed. Frequency analysis was performed by a hard wired frequency analyser (Medelec FA 66). This instrument gave a histogram display of the amplitude of the acceleration for twelve logarithmically spaced frequencies between 2 and $20 \mathrm{~Hz}$. Recordings also made use of a Neurolog electronic averager.

Some observations were made of patients in an anaesthetic room of a hospital, all the other studies were con- ducted in a basement laboratory where there was little structure vibration. Apart from the patients the subjects were medical staff and professional physiologists. Informed consent was obtained in each case and the study had ethical approval. None of the subjects had a conspicuously large tremor.

\section{Results}

Active muscular contraction: horizontal plane

These observations were made using the treme orograph. When the fingers were held partially extended by a minor voluntary effort some tremor was always visible to the naked eye; most subjects were previously unaware that they had such a tremor (fig 2). The tremor did not consist of a single rhythm; examination of the records suggested that a wide range of frequencies was present. The frequency analyser confirmed the existence of a broad spectrum of frequencies, with, usually, a single peak (fig 7). This is not the type of activity to be predicted by the servo theory where a regular oscillation at a frequency determined by the time delay in the neuro-muscular circuit should be found.

When the hand was tapped manually or by the rotating brush a series of up to five decrementing oscillations resulted. The frequency of these oscillations was similar to the frequency of the spectral peak of the spontaneous tremor. For five subjects (three women, two men) the frequency of the transients following a tap was $9.3 \pm 1.2 \mathrm{~Hz}$ (mean and SD). The frequency peak of the spontaneous tremor was $10.0 \pm 1.6 \mathrm{~Hz}$. Use of the $t$ test showed that there was no significant difference between these figures. When inertia was added to the instrument both frequencies were slowed to a similar extent. One author (ML) was tested on 10 separate days and the two sets of figures are closely related (fig 3 ). The correlation was very good; the calculated figure for the correlation coefficient being 0.98 . Others ${ }^{1}$ have noted a very close relationship between the power spectra of damped oscillations of the hand caused by taps and spontaneous tremor. It is accordingly a reasonable assumption that the spectral peak of the spontaneous

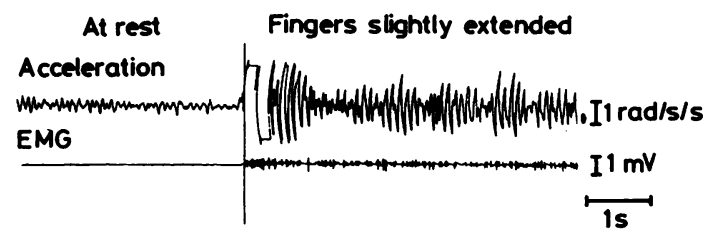

Fig 2 Tremor at rest and with partial finger extension; there is a large increase. The aperiodic nature of the tremor is evident. Such a result is in accordance with an asynchronous mechanical input rather than an oscillation in a neuromuscular servo. 


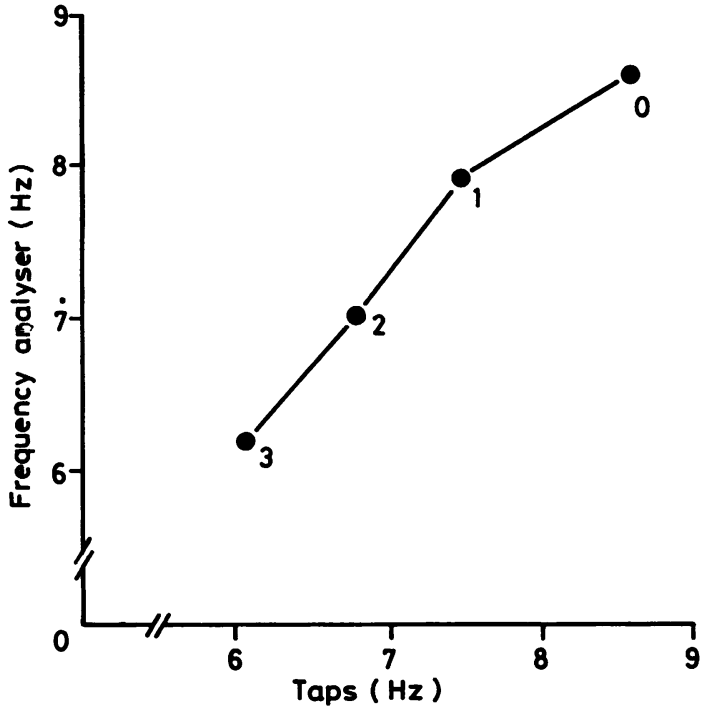

Fig 3 The close relationship between the frequency of the transients following $a$ tap and the frequency of the peak in the tremor spectrum as indicated by the frequency analyser. The figures by the points $(0,1,2,3)$ refer to the added inertia $\left(\mathrm{g} \mathrm{m}^{2}\right)$. Added inertia always slowed the transients and decreased the frequency of the spectral peak.

tremor is due to the underdamped resonance revealed by tapping.

The cause of this resonance is fundamental to an understanding of tremor mechanisms and in this context the EMG data are germane. When the fingers are partially extended the area of active musculature of the extensor digitorum communis could be discerned from the changes in the contour of the forearm, and it was over this area that our electrodes were placed. In eight subjects we made observations during repeated

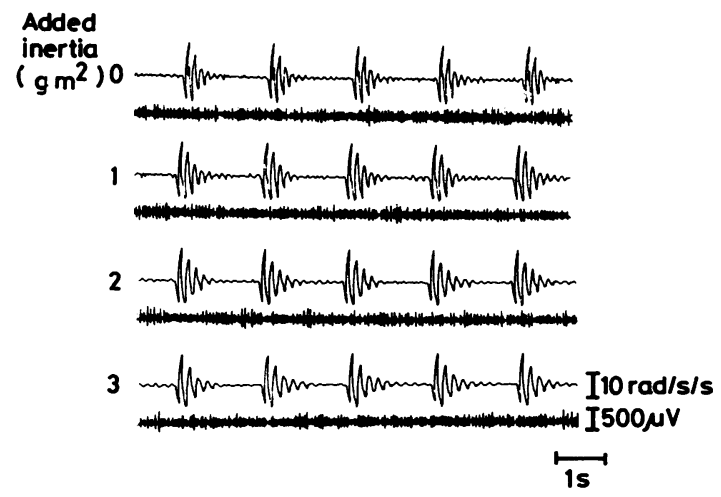

Fig 4 Responses to taps with the fingers partially extended. There is tonic activity in the EMG which shows no modulation during the oscillatory transients. Added inertia slows the transients. taps both without added inertia and with increases of 1,2 , and $3 \mathrm{~g} \mathrm{~m}^{2}$. We never saw any modulation of the EMG even when the size of the taps was adjusted to produce a peak acceleration many times the level of the resting tremor (fig 4). Listening to the EMG on a loudspeaker, and integrating it also failed to reveal any modulation. As it seemed possible that we were missing a minor effect the rectified EMG was averaged on 17 occasions. The averager was triggered by the initial movement of the transient and 16,32 , or 64 sweeps of 1 s duration were used. On 14 occasions there was no added inertia, on the three others the inertia was raised by $2 \mathrm{~g} \mathrm{~m}^{2}$. No modulation of the EMG was detected (fig 5). The motor units active in these experiments were presumably small and tonic; we must conclude that they are not connected into stretch reflex circuits, at least with the levels of force used here. None of the experiments undertaken in the horizontal plane where the weight of the limb is supported and the degree of muscular activity is minimal produced any evidence in favour of the servo-loop hypothesis of tremor generation. We concluded that some alternative explanation of the mechanical transients should be sought and have re-examined some of the other evidence claimed to support the "servo" hypothesis.

\section{Electrical stimulation and frequency analysis of the EMG}

Ischaemia reduces the amplitude of physiological tremor and this finding has been interpretated in favour of the servo theory, the alteration being attributed to changes in the muscle spindles. ${ }^{3}$ We have reinvestigated this by applying repetitive electrical stimuli to the motor point of extensor digitorum communis and recording the motion with the tremorograph. In the relaxed subject weak shocks at $1 \mathrm{~Hz}$ or less generated a series of oscillatory transients essentially similar to those produced by taps discussed below. Ischaemia for $3 \mathrm{~min}$ produced by a cuff round the upper arm did not reduce these transients which we attribute to the muscular twitch disturbing the underdamped second order system. With shocks at a higher

$$
\text { Control Added inertia }\left(2 \mathrm{~g} \mathrm{~m}^{2}\right)
$$

Averaged rect. EMG

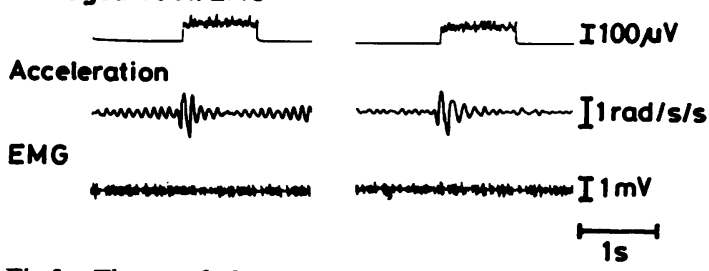

Fig 5 The rectified EMG has been averaged over a $1 \mathrm{~s}$ period. There is no modulation. 16 cycles were averaged. 
Control 2 min occlusion Cuff off ( $30 \mathrm{~s}$ )

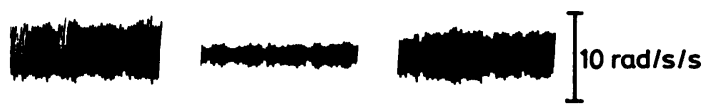

Stimulation at $8 \mathrm{~Hz}$

Fig 6 Exaggerated tremor resulting from electrical stimulation of extensor digitorum communis. Ischaemia causes a substantial reduction in the tremor. Recovery follows deflation of the cuff.

frequency a resonance was found between 8 and $11 \mathrm{~Hz}$. At this frequency the strength of the shocks was adjusted to give an exaggerated but not large tremor. Application of the shocks for some minutes showed no diminution in the size of the tremor. In seven experiments on four subjects ischaemia greatly reduced the tremor. The diminution was sometimes clear after $20 \mathrm{~s}$ and was always marked after $2 \mathrm{~min}$. Following deflation of the cuff there was recovery in the size of the tremor which was always rapid but sometimes incomplete (fig 6). On three occasions the flexor musculature was stimulated with corresponding results. We attribute these effects to muscular fatigue; fatigue reduces the force of muscular contraction and lengthens the duration of a twitch as has long been known. ${ }^{8}$ These effects will reduce the vigour of the perturbations put into the system by muscular action. It is highly unlikely that the muscle spindles were contributing in our experiments. We believe that fatigue may explain the reduction of spontaneous tremor that is caused by ischaemia.

When the rectified EMG was frequency analysed the spectral peak was always at or near the upper end of the analyser's range $(20 \mathrm{~Hz})$. For one subject (tested nine times) the average peak voltage was at

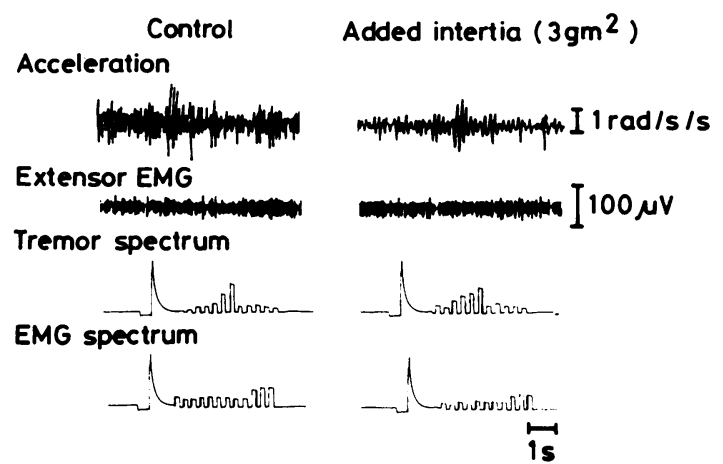

Fig 7 Frequency analysis of the mechanical tremor and of the rectified EMG. The frequency analyser displays an exponentially decaying epoch marker then histogram bars at the following frequencies $(\mathrm{Hz}) 2 \cdot 0,2 \cdot 5,3 \cdot 1,3 \cdot 75,4 \cdot 6,5 \cdot 7$, $7 \cdot 0,8 \cdot 7,10 \cdot 7,13 \cdot 2,16 \cdot 2$, and 20 . In this example the mechanical spectrum peaks at $7.0 \mathrm{~Hz}$ and the EMG at about $16 \mathrm{~Hz}$.
$16.0 \pm 2.0 \mathrm{~Hz}$, a figure very different from the mechanical peak at $8.6 \pm 0.9 \mathrm{~Hz}$ (fig 7). Whilst added inertia always reduced tremor frequency it did not significantly affect the demodulated EMG peak frequency. One subject was tested without extra inertia and also with the addition of $1,2,3,4$, and $5 \mathrm{~g} \mathrm{~m}^{2}$. The frequency of the EMG peak was $17 \cdot 3 \pm 2 \cdot 0 \mathrm{~Hz}$ (mean and SD). The correlation with inertia was negligible (correlation coefficient $=-0.05$ ). The frequency peak of the mechanical spectrum was $7.0 \mathrm{~Hz}$ with no added inertia, reducing to $5.7 \mathrm{~Hz}$ with $5 \mathrm{~g} \mathrm{~m}^{2}$ added. Stiles ${ }^{9}$ also investigated the demodulated EMG and found that with small amplitude tremors as seen in the unfatigued hand there was no spectral peak in the $8-12 \mathrm{~Hz}$ region. The findings confirm the absence of significant grouping of EMG activity at the principal tremor frequency.

\section{Oscillation at rest}

The minor effort required from the subjects in the experiments was eliminated when the subjects relaxed and allowed their fingers to come to the semiflexed position of rest. At this time the EMG of both the flexors and extensors was quiescent. Clinical electromyographers appear to be agreed that in normal subjects there are no EMG discharges in relaxed limbs. ${ }^{10}$ In this situation there was usually no tremor visible to the naked eye but tremor was clearly seen in the tremorograph recording. Taps delivered manually or by the rotating brush again always induced a series of decrementing oscillations (fig 8). A logarithmic amplifier showed that the fall was exponential as would be expected of a system with predominantly viscous damping. The frequency of the spontaneous tremor was again similar to that of the transients induced by tapping. The same five subjects mentioned earlier gave values of $9.2 \pm 2.0 \mathrm{~Hz}$ for the tremor peak and 9.0 $\pm 1.4 \mathrm{~Hz}$ for the transients. Both values

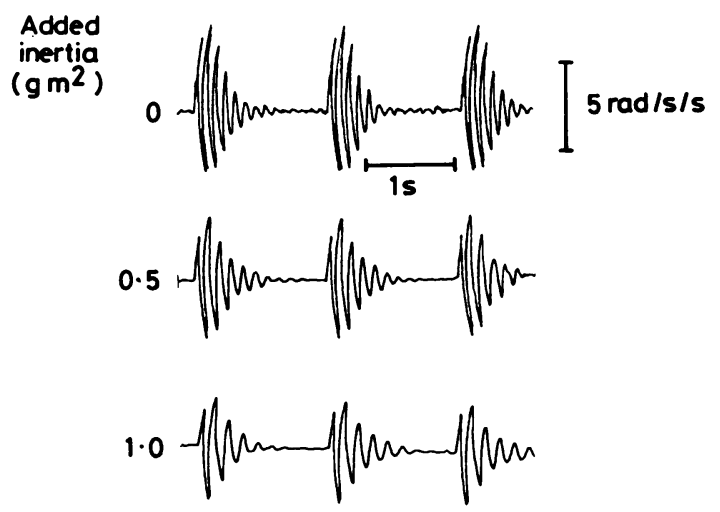

Fig 8 Oscillation transients following taps in a relaxed subject. Added inertia again slows the oscillations. 
were reduced systematically when inertia was added. When the figures for one subject (tested on $10 \mathrm{occa}-$ sions) were plotted a close relationship was again seen. For the mean values with added inertia of 0,1 , 2 , and $3 \mathrm{~g} \mathrm{~m}^{2}$ the correlation coefficient was 0.99 . Here, as with the tensed hand, the evidence is compelling that the frequency characteristics of the tremor are mainly determined by the resonance revealed by the taps. We consistently noted that the waveform became simpler when inertia was added and while the frequency peak in the spectrum fell it became more sharply tuned. These effects are readily explicable on the basis of equation II (below) which defines the increased " $Q$ " as inertia rises. The servo theory postulates that feedback becomes positive at the tremor frequency, but it is clear that positive feedback applied to a mechanically underdamped system would result in a wild uncontrollable motion. Brown, Rack \& Ross $^{11}$ concluded that the stretch reflex would not support a $8-11 \mathrm{~Hz}$ tremor of the thumb because the timing was inappropriate.

The wrist can be regarded as a torsion pendulum, and, because the frequency of the transients did not change as the oscillations became smaller, it has sensibly linear characteristics. If equation I (below) is applicable a linear relationship should exist between

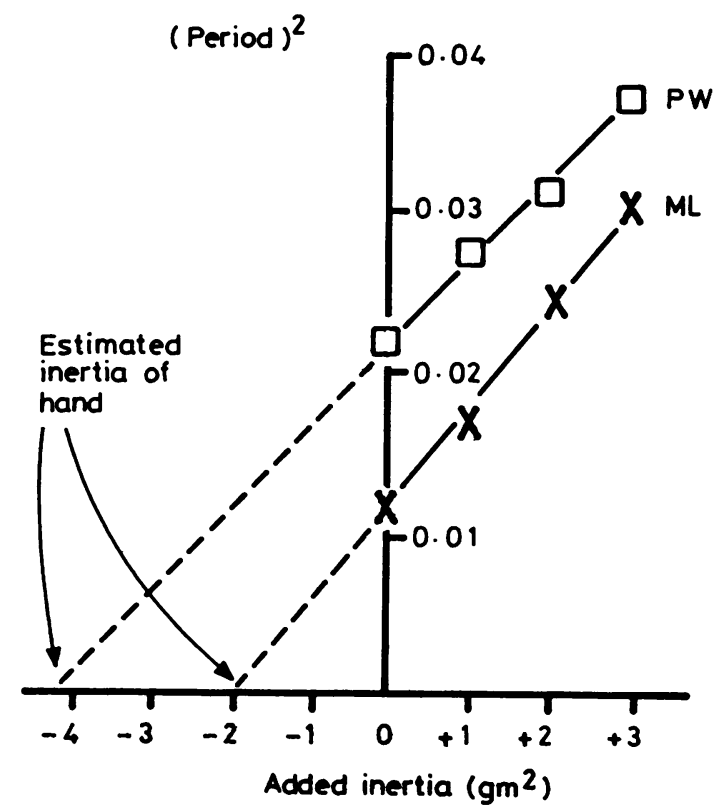

Fig 9 Linear relationship between added inertia and period squared in two men. P.W. has an unusually large hand. The less the inertia the shorter the period. The extrapolation to the abscissa indicates the inertia which would have to be subtracted from the hand to reduce the period to zero; it thus gives the inertia of the hand itself. added inertia $(\mathrm{J})$ and the square of the period of the oscillation $\left(f^{-2}\right)$. The results with two subjects are shown in figure 9 . The data fell essentially on straight lines as predicted. By extrapolation of the lines to the $\mathrm{X}$ axis the original inertia of the hand may be ascertained; the value for $\mathrm{ML}$ is $1.8 \mathrm{~g} \mathrm{~m}^{2}$. $\mathrm{PW}$ is a large heavily built man with an unusually large hand; his value is about $4.2 \mathrm{~g} \mathrm{~m}^{2}$. The moment of inertia for geometrically similar structures varies with the fifth power of a linear dimension so a wide scatter of values for different subjects is characteristic of the data. The frequency of the transients for ML without added inertia was $9 \cdot 2 \mathrm{~Hz}$; by the use of equation I the stiffness of the forearm musculature is calculated: $6.0 \mathrm{~N} \mathrm{~m} / \mathrm{rad}$. For PW the frequency of the transients was $6.7 \mathrm{~Hz}$ giving a stiffness value of $7 \cdot 1 \mathrm{~N} \mathrm{~m} / \mathrm{rad}$. As would be expected from scaling considerations ${ }^{12}$ muscle stiffness varies much less than inertia.

The spontaneous tremor waves could often be seen to be related to the heartbeat. In one subject the occasional extrasystole was associated with a change in the envelope of the tremor waveform (fig 10). In five subjects the contribution of cardiac action to the tremor was evaluated by triggering an averager from the QRS complex of the ECG. In every instance it was found that a significant proportion of the tremor was related to the heart beat; this was true even when the relationship was not immediately apparent to the naked eye. The cardiac component sometimes had a complex waveform. Three of the subjects were tested with the following amounts of added inertia $-0,1,2$, $3,4,5,6,7$, and $8 \mathrm{~g} \mathrm{~m}^{2}$. With the added inertia the relationship to the heart beat was found often with added clarity although the frequency of the oscillations was decreased. In each of these experiments an average was taken under normal conditions and with the circulation to the arm arrested by a cuff. Arterial occlusion made little or no difference to the cardiac component of the tremor. A figure illustrating these results has previously been published. ${ }^{13}$ When the

$$
\text { Control } \quad 1.5 \mathrm{~g} \mathrm{~m}^{2}
$$

\section{Acceleration}

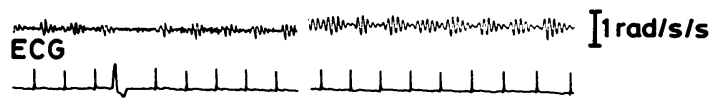

Frequency analyser

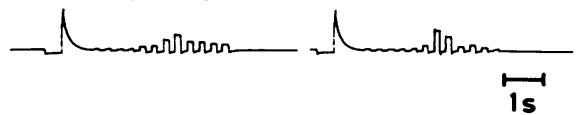

Fig 10 In the control record the tremor waves are most obvious at about the time of the T wave of the ECG, but the relationship is "noisy". A conspicuous alteration in the pattern follows the extrasystole. With added inertia there is a reduction in the high frquency components of the tremor and the relationship of the envelope to the heartbeat is obvious. 
fingers were voluntarily extended the cardiac component remained; its size was little changed but it came to represent a much smaller proportion of the total tremor. Other workers have noted similar relationships. ${ }^{14}$ We believe that the tremor encountered when the muscles are inactive is due to the mechanical drive from the ballistocardiogram and other minor influences such as respiratory movements and distant muscular action exciting the underdamped resonant system.

\section{The anaesthetised and paralysed state}

These observations were made on six women who were being anaesthetised and given full doses of neuromuscular blocking drugs to prepare them for surgical operations. A figure illustrating these results has been published. ${ }^{15}$ The response to taps was similar when conscious and after anaesthesia and paralysis: control $8.3 \pm 1.4 \mathrm{~Hz}$ and $8.8 \pm 0.8 \mathrm{~Hz}$ after anaesthesia and paralysis. Use of the $t$ test showed no significant difference. The effect of adding a bar of $1.0 \mathrm{~g} \mathrm{~m}^{2}$ inertia was to reduce the frequency of the transients and to $5.5 \pm 2.4 \mathrm{~Hz}$ before anaesthesia and $6.0 \pm 0.6 \mathrm{~Hz}$ when anaesthetised and paralysed. We also observed a physiological tremor in the paralysed state which was similar to that seen in conscious subjects at rest. This is conclusive evidence that the resonant behaviour of the relaxed wrist is not due to neurological circuits but is a passive property of the tissues.

\section{Taps in the vertical plane}

When the hand was held horizontal there was continuous and apparently asynchronous activity in the extensor digitorum communis. With moderately vigorous downward blows delivered by a tendon hammer a phasic stretch reflex was seen followed by a silent period, but any further modulation was exceptional. When the hand was coupled to the motor (methods) the maximal force available was less $(0.26 \mathrm{~N} \mathrm{~m})$ and the phasic stretch reflex and silent period were sometimes seen but were less conspicuous. With smaller forces the modulation was even less clear; with forces of $0.026 \mathrm{Nm}$ there was no modulation of the EMG even though the taps caused transient oscillations similar to those seen with the tremorograph and substantially larger than the spontaneous tremor.

Experiments on 12 occasions (eight subjects) were undertaken to ascertain the effects of fatigue. In a typical experiment the subject supported a weight by exerting a torque of about $0.3 \mathrm{Nm}$ for $5 \mathrm{~min}$. Observations made immediately after the weight was removed often showed an increased number of oscillatory transients following a tap from the motor. Furthermore, this increased response was associated with modulation of the EMG, a series of grouped dis-

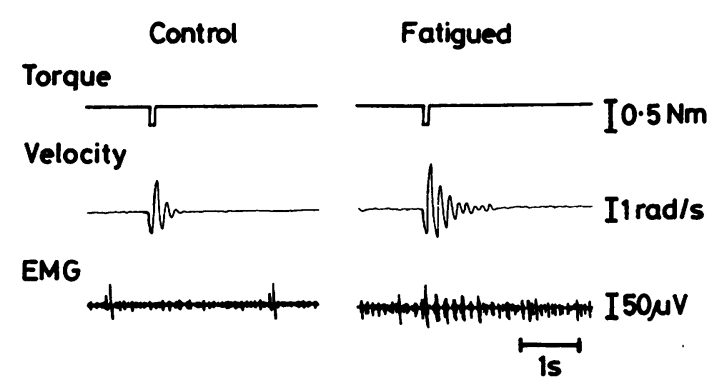

Fig 11 Observations in the vertical plane with the hand held horizontal. A downward pulse of torque is applied by a motor. In the control period the responses were very stereotyped. Fatigue was then induced by applying a weight to the hand for five minutes. Afterwards, the number of waves were increased and the EMG was then modulated.

charges being seen (fig 11). Occasionally the amplitude of the oscillations would increase transiently after the initial decrease. These rhythmic responses could sometimes be enhanced by minor degrees of co-contraction of the forearm musculature. These effects were transient persisting for less than $3 \mathrm{~min}$. Immediately after the removal of the weight there was commonly an increase (sometimes large) in the level of spontaneous tremor. The grouping of EMG discharges seen in the fatigued muscle with forces significantly larger than those involved in norma tremor are reminiscient of the records of Lippold. ${ }^{3}$

\section{Discussion}

In earlier work we have shown that for large and moderate forces the wrist is resonant at a frequency of about $2 \mathrm{~Hz}$, but as the force is reduced there is a considerable rise in resonant frequency reaching about $8 \mathrm{~Hz}$ with the smallest values used. We have attributed this to stiffening of the postural system that occurs in the absence of movement: postural thixotropy. ${ }^{16}$ The formula for a torsion pendulum is:

$$
\mathrm{f}=\frac{1}{2 \pi} \sqrt{\frac{\mathrm{K}}{\mathrm{J}}}
$$

where $f$ is the resonant frequency, $K$ is the stiffness and $\mathrm{J}$ the inertia. Accordingly, a fourfold rise in resonant frequency corresponds to a sixteenfold increase in stiffness as the inertia can be assumed to remain constant. Short range stiffness of isolated muscle was described by D K Hill. ${ }^{17}$ The sharpness of tuning, or liability to resonate, of such a system is given by the equation:

$$
\mathrm{Q}=\frac{1}{\mathrm{c}} \sqrt{\mathrm{KJ}}
$$

where $\mathrm{Q}$ is the sharpness of tuning and $\mathrm{c}$ is the damp- 
ing constant. As the system stiffens (increased $\mathrm{K}$ ) $\mathrm{Q}$ will rise. It is to this that we ascribe the clearly underdamped nature of the passive wrist for small movements.

We have established that for small movements the forearm and hand form an underdamped system, the resonant frequency of which can be lowered by added inertia. When tapped a series of decrementing transients are established. The frequency of a damped vibration is lower than the resonant frequency but for light damping the effect is quite small. ${ }^{18}$ Even in fully relaxed subjects with no recordable muscular activity there is a spontaneous tremor which has the characteristics to be expected of such a system subjected to minor mechanical disturbances. One such disturbance is clearly the ballistocardiogram. It may be assumed that other small movements such as respiratory activity will also cause the system to ring at its resonant frequency.

In most of our experiments where the muscles were active there was no evidence of grouped EMG activity. In Lippold's ${ }^{3}$ experiment the finger was abruptly prodded. Stimulation of the skin of the finger has been shown to have profound effects on the discharge of motor neurons supplying the hand. ${ }^{19}$ To quote these authors "of the various regions tested on the fingers, the tip was by far the most powerful source of reflex activity". Cutaneous activity has also been shown to play a significant role in the servo control of the thumb. ${ }^{20}$ The nail bed is richly innervated and tapping the finger tip under conditions in which there is no stretching of muscles can change muscular reflexes. ${ }^{21}$ These factors or idiosyncrasies of the subjects may account for the difference in the results. Furthermore, in Lippold's experiment and in those of Young and Hagbarth ${ }^{22}$ the fingers or hand plus fingers were held outstretched and the resultant fatigue may have been significant. Both Lippold and Hagbarth and Young sometimes deliberately enhanced tremor by fatiguing the muscles before making their observations.

When we studied vertical movement with fatigued muscles modulation of the EMG with taps was clearly seen. The striking effects of fatigue on tremor have been investigated by Stiles. ${ }^{9}$ He concluded that a visco-elastic mass mechanism primarily determined the small amplitude tremor while the large displacement tremors seen after fatigue involved neural feedback. Similar considerations may explain the finding that there was a sharp peak at $10 \mathrm{~Hz}$ in the spectrum of the demodulated EMG in unconstrained tremor. ${ }^{7}$ Alternatively this finding could be explained if there was a single large motor unit firing at this frequency near to the recording site. Sanes ${ }^{23}$ has studied tremor in patients with loss of large proprioceptive fibres. With fatigue, although instability increased, a rhyth- mic enhanced tremor was not seen. He concluded that enhanced physiological tremor was dependent on somaesthetic afferent input.

With active muscular contraction the discontinuous mechanical input produced by unfused contractions and applied to a resonant system must play a major role in the generation of tremor. We agree with Stiles and Randall ${ }^{6}$ that "the price of freedom of movement, in a system having continuous forcing is a certain amount of instability". When the forcing input is random with power distributed uniformly throughout the frequencies under consideration the peak frequency of the tremor will be identical with the resonant frequency. If the power spectrum of the input is not that of "white noise" identity cannot be expected. Cooling slows physiological tremor ${ }^{3}$ but the effect may be due to the slower contraction and relaxation times of cooled muscle; this slowing will shift the spectrum of the forcing input emphasising the lower frequencies.

We conclude that the most economical explanation for the physiological tremor that we have studied is that the mechanical properties of the postural system produce an inbuilt instability which makes small oscillations inevitable. No special activity of the nervous system is required to maintain these oscillations which will occur whether the input is from the muscles, ballistocardiogram, or applied taps. There may however be synchronised activity in the nervous system when the muscles are fatigued or with pathological tremors. Under isometric conditions the stiffness of the system (K, equation I) will be greatly increased and the resonant frequency accordingly elevated. It has been noted that with mild contractions the spectral peak near $10 \mathrm{~Hz}$ is indeed then commonly absent. ${ }^{7}$ If strong forces are exerted under similar conditions the peak may reappear and other explanations, such as Renshaw cell rebound, must then be sought. ${ }^{24}$

The authors thank Dr DB Scott for facilitating the observations made under anaesthesia, the patients, and the subjects.

\section{References}

${ }^{1}$ Rietz RR, Stiles RN. A viscoelastic-mass mechanism as a basis for normal postural tremor. J Appl Physiol 1974;37:(6) 852-60.

${ }^{2}$ Marshall J, Walsh EG. Physiological Tremor. J Neurol Neurosurg Psychiatry 1956;19:260-7.

${ }^{3}$ Lippold OCJ. Oscillation in the stretch reflex arc and the origin of the rhythmical 8-12 c/s component of physiological tremor. J Physiol (Lond) 1973;206:359-82.

${ }^{4}$ Marsden CD, Meadows JC, Lange GW, Watson RS. Effect of deafferentation on human physiological 
tremor. Lancet 1967;ii:700-2.

${ }^{5}$ Wood WS, McNaull J. A simple method of recording angular acceleration. The Engineer 1962;2213:569-70.

${ }^{6}$ Stiles RN, Randall JE. Mechanical factors in human tremor frequency. J Appl Physiol 1967;23 (3):324-30.

${ }^{7}$ Burne JA, Lippold OCJ, Prior M. Proprioceptors and normal tremor. J Physiol (Lond) 1984;348:559-72.

${ }^{8}$ Marey EJ. Animal Mechanism London: King 1874 Fig 4 p 34.

${ }^{9}$ Stiles RN. Frequency and displacement amplitude relations for normal hand tremor. J Appl Physiol 1976;40 (1):44-54.

${ }^{10}$ Basmajian JV. Muscles Alive-Their Functions Revealed by Electromyography. Baltimore: Williams \& Wilkins, 1967.

${ }^{11}$ Brown TIH, Rack PMH, Ross HF. Different types of tremor in the human thumb. J Physiol (Lond) 1982; 332:113-23.

12 McMahon TA. Muscles, Reflexes and Locomotion. New Jersey: Princeton University Press, 1984.

${ }^{13}$ Lakie M, Walsh EG, Wright GW. On tuning a physiological tremor. J Physiol (Lond) 1983;334:32P.

${ }^{14}$ Marsden CD, Meadows JC, Lange GW, Watson RS. The role of the ballistocardiac impulse in the genesis of physiological tremor. Brain 1969;92:647-62.

${ }^{15}$ Lakie M, Scott DB, Walsh EG, Wright GW. Resonance at the wrist in anaesthetised subjects. $J$ Physiol (Lond) 1983;338:32P.
${ }^{16}$ Lakie M, Walsh EG, Wright GW. Resonance at the wrist demonstrated by the use of a torque motor: an instrumental analysis of muscle tone in man. $J$ Physiol (Lond) 1984;353:265-85.

${ }^{17}$ Hill DK. Tension due to interaction between the sliding filaments in resting striated muscle. The effect of stimulation. J Physiol (Lond) 1968;199:637-84.

${ }^{18}$ Burton R. Vibration and Impact. New York: Dover. 1968:50.

${ }^{19}$ Caccia MR, McComas AJ, Upton ARM, Blogg T. Cutaneous reflexes in the small muscles of the hand. $J$ Neurol Neurosurg Psychiatry 1973;36:960-77.

${ }^{20}$ Marsden CD, Merton PA, Morton HB. The sensory mechanism of servo-action in human muscle. J Physiol (Lond) 1977;265:521-35.

${ }^{21}$ Garnett R, Stephens JA. The reflex responses of single motor units in human first dorsal interosseus muscle following cutaneous afferent stimulation. $J$ Physiol (Lond) 1980;303:351-64.

${ }^{22}$ Young RR, Hagbarth K-E. Participation of the stretch reflex in human physiological tremor. Brain 1979;102: 509-26.

${ }^{23}$ Sanes JN. Absence of enhanced physiological tremor in patients without muscles or cutaneous afferents. $J \mathrm{Neu}$ rol Neurosurg Psychiatry 1985;48:645-9.

${ }^{24}$ Elble RJ, Randall JE. Motor unit activity responsible for the 8-12 $\mathrm{Hz}$ component of human physiological finger tremor. J Neurophysiol 1976;39 (2):370-83. 